\title{
Long-Term Outcome of Preoperative Disrupted Inner/Outer Segment Junctions Assessed Using Spectral-Domain Optical Coherence Tomography in Patients with Idiopathic Epiretinal Membrane
}

\author{
Maiko Inoue Akira Arakawa Shin Yamane Kazuaki Kadonosono \\ Department of Ophthalmology, Yokohama City University Medical Center, Yokohama, Japan
}

\section{Key Words}

Epiretinal membrane $\cdot$ Spectral-domain optical coherence tomography $\cdot$ Inner/outer segment junction $\cdot$ Lamellar macular hole

\begin{abstract}
Purpose: To evaluate the anatomic and functional outcomes as assessed by spectral-domain optical coherence tomography in patients with a preoperative disrupted or irregular photoreceptor inner/outer segment (IS/OS) junction who underwent idiopathic epiretinal membrane (ERM) surgery and were followed for at least 2 years. Methods: We retrospectively studied all of the patients with idiopathic ERM who had a preoperative disrupted IS/OS junction and were scheduled to undergo a transconjunctival 25-gauge vitrectomy between September 2007 and October 2009. All of the patients who had a 2-year or longer follow-up period were included in the study. The best-corrected visual acuity (BCVA) and structural changes in the IS/OS junction were examined before and 3, 6, 12, and 24 months after surgery. Furthermore, patients were divided into two groups based on whether the preoperative disrupted IS/OS junction had been caused by a lamellar macular hole (LMH type) or swelling as a result of ERM traction (S type). Morphologic changes in the
\end{abstract}

IS/OS junctions of each type were also investigated. Results: A total of 17 patients were identified in this study. Overall, a significantly better postoperative BCVA was seen after surgery; in particular, the BCVA at 24 months was significantly better than that at 12 months $(p=0.018)$. Although the disrupted IS/OS junction appeared normal in 1 out of 17 eyes at 12 months, 7 eyes appeared normal at 24 months. These 7 eyes showed significant postoperative improvement in BCVA compared with the remaining 10 eyes $(p=0.045, p=$ $0.021, p=0.019$, and $p=0.015$ at $3,6,12$, and 24 months, respectively). The preoperative disrupted IS/OS junction was classified as LMH type in 8 eyes and as $\mathrm{S}$ type in 9 eyes. The LMH type, rather than the $\mathrm{S}$ type, tended to show a persistently disrupted IS/OS junction at 24 months, although the difference was not significant. Conclusion: Anatomic and functional recovery of preoperative disrupted IS/OS junctions can be acquired even after more than 1 year postoperatively. However, eyes with persistent irregularities of the IS/OS junction at a 2-year follow-up, especially those with LMH type irregularities, might have a limited visual improvement.
Copyright $\odot 2012$ S. Karger AG, Basel

\section{KARGER \\ Fax +4161306 1234 \\ E-Mail karger@karger.ch}

www.karger.com (c) 2012 S. Karger AG, Basel

$0030-3755 / 12 / 2284-0222 \$ 38.00 / 0$

Accessible online at:

www.karger.com/oph
Maiko Inoue, $\mathrm{MD}$

4-57 Urafune-cho, Minami-ku

Yokohama, Kanagawa 232-0024 (Japan)

Tel. +81 452615656

E-Mailmaicoo@urahp.yokohama-cu.ac.jp 


\section{Introduction}

Surgical correction of the idiopathic epiretinal membrane (ERM), first reported by Machemer [1] in 1978, is useful for improving vision. However, some cases show poor visual recovery even after complete removal of the ERM $[2,3]$. Several factors, such as preoperative visual acuity [3], the duration of the symptoms before surgery [2], and the presence/absence of cystoid macular edema [4], have been suggested to be prognostic factors influencing postoperative visual acuity. Recently, since the introduction of spectral-domain optical coherence tomography (SD-OCT) as a useful tool for the noninvasive diagnosis and monitoring of macular diseases, including ERM [5], investigators have suggested that the appearance of the photoreceptor layer may be associated with postoperative visual acuity [6-9].

We previously reported that an intact photoreceptor inner/outer segment (IS/OS) junction, as assessed preoperatively, was strongly associated with better postoperative visual acuity and better visual recovery than a disrupted or irregular IS/OS junction. Furthermore, none of the eyes with a preoperative disrupted IS/OS junction showed structural recovery of the IS/OS junction after a 1-year follow-up period [8]. However, no studies have reported the long-term anatomical outcomes of IS/OS junctions in patients with idiopathic ERM.

The purpose of this study was to use SD-OCT to evaluate the long-term follow-up observations of preoperative disrupted or irregular IS/OS junctions in patients who had undergone ERM surgery. The anatomic features, as assessed using SD-OCT, were followed until at least 2 years after surgery, and the functional outcomes were investigated.

\section{Patients and Methods}

We studied all of the patients with idiopathic ERM who had a preoperative disrupted or irregular IS/OS junction and were followed up for 24 months or longer after surgical treatment at the Yokohama City University Medical Center between September 2007 and October 2009. Patients with eye diseases influencing visual acuity, such as glaucoma, macular degeneration, diabetic retinopathy, or rhegmatogenous retinal detachment, were excluded. In this study, cases with a preoperative intact IS/OS junction were also excluded because such eyes have been reported to exhibit an intact IS/OS junction with sufficient visual recovery at a 1-year follow-up examination [8]. The study was conducted according to the tenets of the Declaration of Helsinki. Informed consent for the research protocol and the data collection was obtained from all of the eligible patients. The ethics committee of Yokohama City University Medical Center approved this retrospective study.

Long-Term Outcome of Preoperative

Disrupted IS/OS Junctions
A 25-gauge pars plana vitrectomy with internal limiting membrane (ILM) peeling was performed by the one retina specialist (K.K.) in all of the cases. Indocyanine green $0.06 \%$ (ICG) was used for staining before removal of the ILM in all cases. The area of ILM peeling was 2-3 optic disk diameters around the fovea. Cataract surgery was combined with vitrectomy in eyes with a clinically significant cataract. Removal of the ERM was defined clinically by ophthalmoscopic and OCT examination.

The best-corrected visual acuity (BCVA) and structural changes in the IS/OS junction based on the SD-OCT findings (Cirrus high-definition OCT; Carl Zeiss, Dublin, Calif., USA) were investigated at $3,6,12$, and 24 months after surgery. The BCVA was converted to the logarithm of the minimum angle of resolution ( $\log$ MAR) equivalent for the statistical analysis. We retrospectively classified the eyes into two groups according to the postoperative structural changes in the IS/OS junction at 24 months after surgery. Visual acuity parameters, such as the BCVA after surgery and the differences between the preoperative and postoperative BCVA after surgery (improvement of the visual acuity) were then compared between the two groups.

Furthermore, patients were also divided into two categories based on whether the preoperative disrupted IS/OS junction originated from a lamellar macular hole (LMH type) or from swelling as a result of ERM traction (S type). The preoperative length of the longest IS/OS junction defect and postoperative morphologic changes in the IS/OS junction were also investigated for each type.

In the present series, SD-OCT was performed for each eye using five-line raster scans in a horizontal way. Scans with a signal strength greater than $7 / 10$ were considered appropriate, and a representative image was selected. Two examiners (M.I. and S.Y.) who were masked to information about the visual acuity of the patients interpreted the macular microstructure in the SD-OCT images together. The photoreceptor layer was imaged as a hyporeflective layer containing a hyperreflective line of IS/OS junction and is bordered by hyperreflective layers of external limiting membrane and retinal pigment epithelium. An intact IS/OS junction was defined as a continuous hyperreflective line. On the other hand, the diagnosis of a disrupted IS/OS was made based on the loss or irregularity of the hyperreflective line corresponding to the subfoveal IS/OS junction.

The Wilcoxon signed-rank test was used to compare preoperative and postoperative visual acuities. The postoperative visual acuity parameters between the two groups were compared using the Mann-Whitney $U$ test. The difference in proportions of the morphology of the normal IS/OS junction at 24 months between the LMH type and the $\mathrm{S}$ type was evaluated using a twosample test for equality of proportions. All of the analyses were conducted using SPSS software (version 17.0 for Windows; Microsoft, Redmond, Wash., USA; SPSS, Inc., Chicago, Ill., USA). p < 0.05 was considered statistically significant.

\section{Results}

The total number of ERM eyes which underwent vitrectomy during this period was 157 . One hundred thirtyfive eyes had preoperative intact IS/OS junctions and 
were excluded from this study. The remaining 22 eyes had preoperative disrupted IS/OS junctions. A total of 17 patients with preoperative disrupted IS/OS junctions who had undergone ERM surgery and had a follow-up period of 2 or more years were included in this study. Five patients were excluded because they had a follow-up period of less than 2 years. The baseline characteristics and clinical data of the patients are shown in table 1 . Of the 17 patients, 4 were men and 13 were women, with the patients ranging in age from 52 to 79 years (mean age 65.9 \pm 7.8 years). The ERM was successfully removed in all cases. Cataract surgery combined with a vitrectomy was performed in 16 eyes with clinically significant cataracts. A vitrectomy alone was performed in 1 pseudophakic eye. There were no complications in any of the cases. Furthermore, there was no recurrence of ERM in the postoperative period.

Overall, the mean $\log$ MAR visual acuity at baseline was $0.56 \pm 0.30$. The mean $\log$ MAR BCVA values at 3 , 6,12 , and 24 months after surgery were $0.34 \pm 0.23,0.31$ $\pm 0.22,0.29 \pm 0.20$, and $0.24 \pm 0.21$, respectively. The postoperative BCVA was significantly improved compared with the preoperative visual acuity $(\mathrm{p}<0.001$, respectively); in particular, the BCVA at 24 months was significantly better than that at 12 months $(\mathrm{p}=0.018)$ (fig. 1).

Regarding IS/OS junction integrity, the disruption of the IS/OS junction at 12 months changed to a normal appearance in only 1 eye. However, 7 eyes had an IS/OS junction with a normal appearance at 24 months (table 2). Among these 7 eyes, the mean $\log$ MAR visual acuity at baseline was $0.62 \pm 0.33$. The mean logMAR BCVA values at $3,6,12$, and 24 months after surgery were $0.30 \pm$ $0.20,0.21 \pm 0.16,0.19 \pm 0.16$, and $0.16 \pm 0.18$, respectively. In the remaining 10 eyes with a persistently disrupted IS/OS junction, the mean logMAR visual acuity at baseline was $0.53 \pm 0.29$. The mean logMAR BCVA values at $3,6,12$, and 24 months after surgery were $0.38 \pm$ $0.25,0.38 \pm 0.23,0.36 \pm 0.20$, and $0.31 \pm 0.25$, respectively. There were no significant differences in postoperative BCVA values between the restored and persistent disruption groups (fig. 1).

On the other hand, the mean differences between the preoperative and postoperative BCVA values at $3,6,12$, and 24 months after surgery were $0.32 \pm 0.22,0.40 \pm$ $0.23,0.43 \pm 0.24$, and $0.46 \pm 0.20$, respectively, in the 7 eyes with the recovered disruptions, and $0.15 \pm 0.19,0.15$ $\pm 0.21,0.17 \pm 0.21$, and $0.23 \pm 0.20$, respectively, in the remaining 10 eyes with persistent disruptions. Thus, the visual acuity of the eyes with recovered disruptions im-
Table 1. Baseline characteristics of all study eyes with ERM having disrupted IS/OS junctions

\begin{tabular}{lc}
\hline Operated eyes & 17 \\
Mean age \pm SD (range), years & $65.9 \pm 7.8(52-79)$ \\
Gender, $\mathrm{n}(\%)$ & \\
$\quad$ Male & $4(23.5)$ \\
$\quad$ Female & $13(76.5)$ \\
Mean baseline logMAR visual acuity \pm SD & $0.56 \pm 0.30$ \\
Preoperative lens status, n (\%) & \\
$\quad$ Phakic, significant cataract & $16(94.1)$ \\
$\quad$ IOL & $1(5.9)$ \\
\hline
\end{tabular}

$\mathrm{IOL}=$ Intraocular lens.

Table 2. Distribution of photoreceptor status before and after idiopathic ERM surgery

\begin{tabular}{|c|c|c|c|c|c|}
\hline \multirow{2}{*}{$\begin{array}{l}\text { Photoreceptor } \\
\text { status }\end{array}$} & \multicolumn{5}{|c|}{ Eyes, n (\%) } \\
\hline & baseline & month 3 & month 6 & month 12 & month 24 \\
\hline Regular & 0 & 0 & 0 & $1(6)$ & $7(41)$ \\
\hline Irregular & $17(100)$ & $17(100)$ & $17(100)$ & $16(94)$ & $10(59)$ \\
\hline
\end{tabular}

proved to a greater degree after surgery $(\mathrm{p}=0.045, \mathrm{p}=$ $0.021, \mathrm{p}=0.019$, and $\mathrm{p}=0.015$; fig. 2).

Of the 17 eyes with disrupted IS/OS junctions, 8 were preoperatively classified as LMH type and 9 were classified as $\mathrm{S}$ type. At 24 months postsurgery, among the 7 eyes with restored normal IS/OS junctions, 2 eyes were $\mathrm{LMH}$ type and the remaining 5 eyes were $\mathrm{S}$ type. On the other hand, among the 10 eyes with persistently disrupted IS/OS junctions, 6 eyes were LMH type and the remaining 4 eyes were $S$ type. The LMH type, rather than the $S$ type, tended to show persistent disruption of the IS/ OS junction at 24 months, although no significant difference was observed $(p=0.101)$. Regarding the LMH type, eyes with a larger preoperative length of the IS/OS junction defect tended to have a persistently disrupted IS/OS junction. On the other hand, the preoperative IS/OS junctions in S type eyes showed diffuse hyporeflectivity; however, a hyperreflective IS/OS junction was gradually restored, and the appearance normalized in 5 eyes. The other $4 \mathrm{~S}$ type eyes continued to exhibit partially hyporeflective IS/OS junctions (table 3 ).

Figures 3 and 4 show examples of changes in $\mathrm{LMH}$ type and $\mathrm{S}$ type eyes, respectively. 


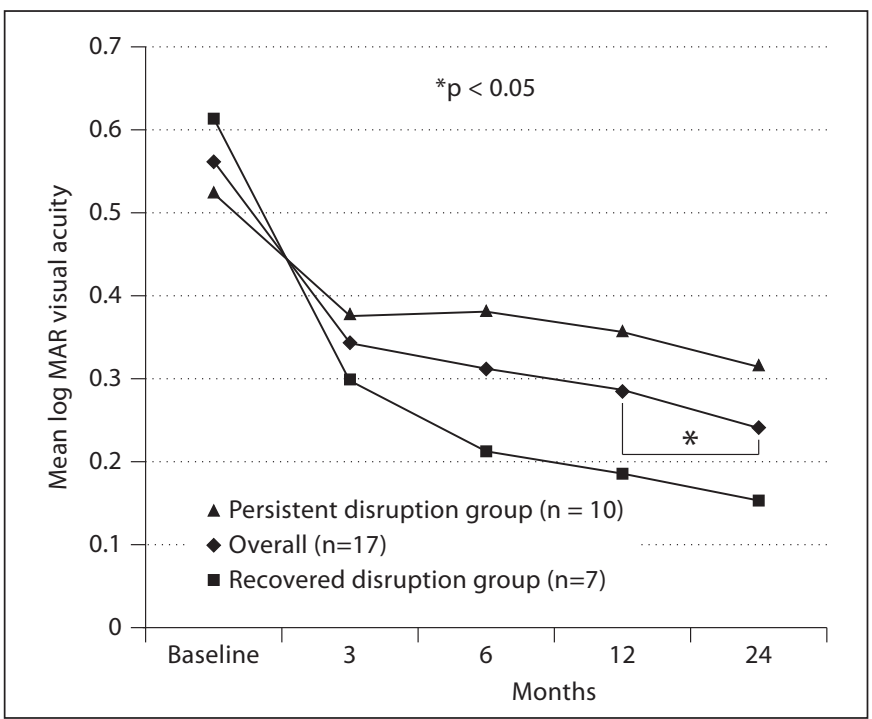

Fig. 1. Changes in mean logMAR BCVA values between baseline and 24 months after surgery overall, in the recovered disruption group, and in the persistent disruption group. The recovered disruption group consisted of patients with an intact IS/OS junction at 24 months after surgery, and the persistent disruption group consisted of patients with a persistently disrupted IS/OS junction at 24 months after surgery. Overall, the postoperative BCVA value improved significantly compared with the preoperative visual acuity ( $p<0.001$, respectively). Significant differences in the changes in logMAR BCVA values at 12 and 24 months after surgery were seen overall $(\mathrm{p}=0.018)$.

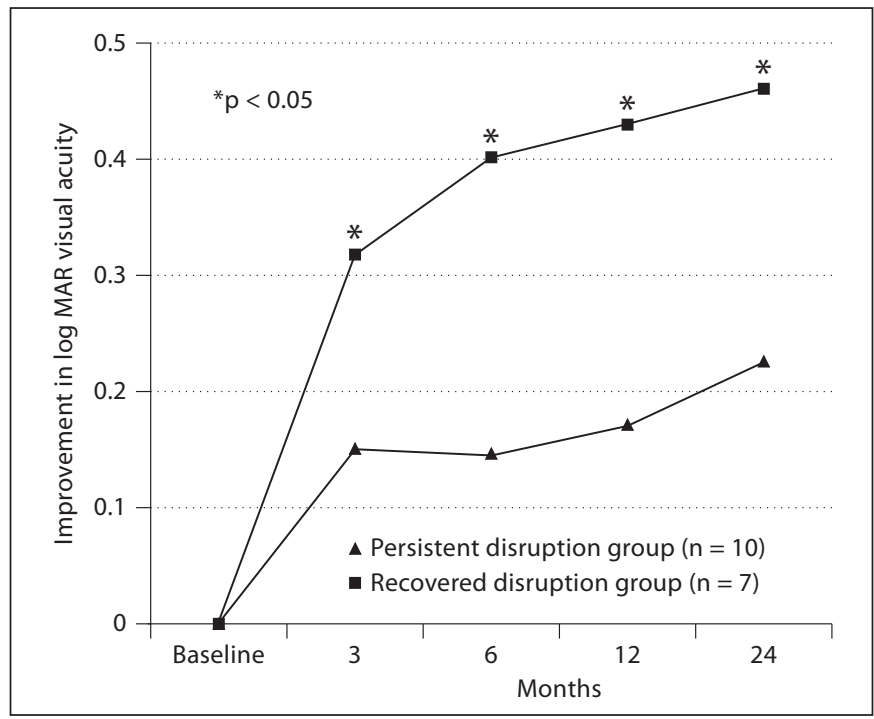

Fig. 2. Graph showing the differences between the preoperative and postoperative BCVA values at 24 months after surgery (improvement in visual acuity) in two groups. The recovered disruption group consisted of patients with an intact IS/OS junction and the persistent disruption group consisted of patients with a disrupted IS/OS junction at 24 months after surgery. The recovered disruption group showed a greater degree of improvement in visual acuity between the baseline value and the postoperative follow-up value $(\mathrm{p}=0.045, \mathrm{p}=0.021, \mathrm{p}=0.019$, and $\mathrm{p}=0.015)$.

Table 3. Baseline characteristics and clinical data before and after ERM surgery





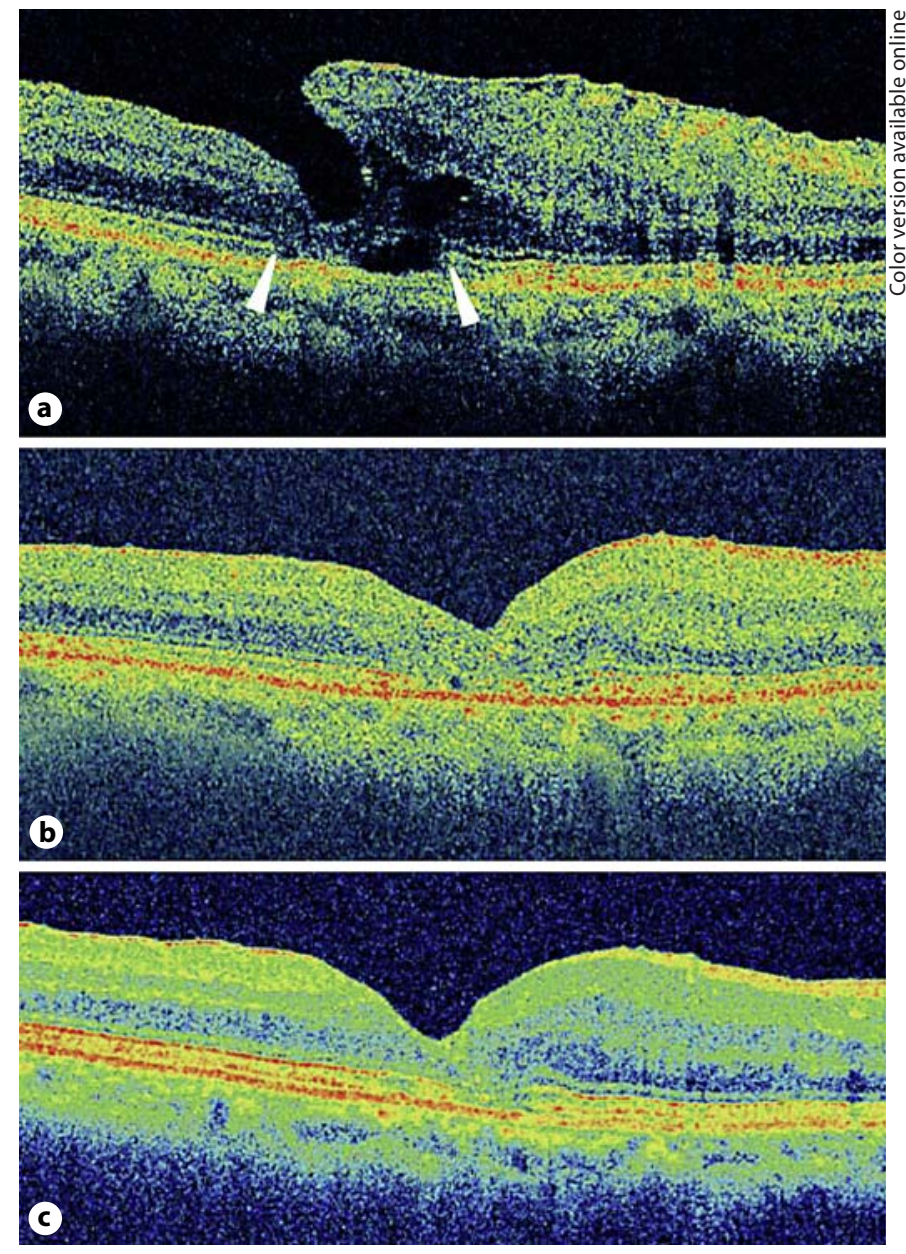

Fig. 3. Patient 10 in table 3: changes in SD-OCT findings between baseline and through 24 months of follow-up in an LMH type eye. a The SD-OCT findings at baseline show an ERM with a non-fullthickness macular hole and a continuous and discontinuous IS/ OS junction, which was evaluated as a disrupted IS/OS junction. The length of the defect in the IS/OS junction was $810 \mu \mathrm{m}$ (arrowheads). The visual acuity was 20/50 in the right eye. b Postoperative OCT at 12 months showed persistent disruption of the IS/ OS junction. c Postoperative OCT at 24 months also revealed persistent disruption of the IS/OS junction. However, at the 2-year follow-up examination, the patient's visual acuity had improved to $20 / 25$.

\section{Discussion}

We previously reported that IS/OS junctions with preoperative disruptions or irregularities continue to exhibit such disruptions or irregularities at one year after surgery [8]. However, the long-term outcomes of these structural changes have not been clearly identified. Furthermore, because preoperative eyes with disrupted IS/OS junctions
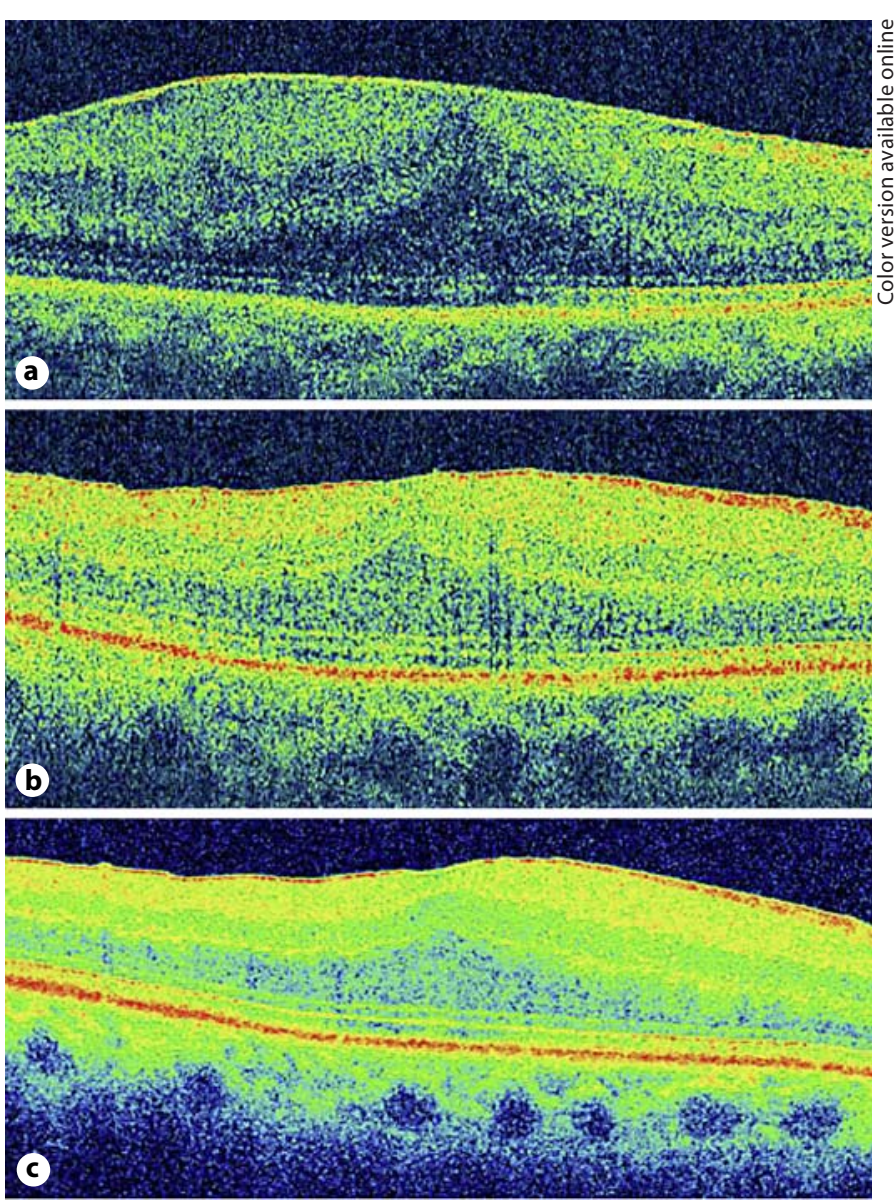

Fig. 4. Patient 6 in table 3: changes in SD-OCT findings between baseline and through 24 months of follow-up in an S type eye. a The SD-OCT findings at baseline show an ERM with a diffuse hyporeflective IS/OS junction; the patient was therefore evaluated as having a disrupted IS/OS junction. The visual acuity was 20/63 in the right eye. b A postoperative OCT at 12 months showed a persistently hyporeflective IS/OS junction. However, the patient's visual acuity had improved to 20/20. c At the 2 -year follow-up examination, the reflectivity of the IS/OS junction had resolved. The patient's visual acuity had stabilized at 20/20. are less common, long-term follow-up assessments have been difficult to perform. This is the first report to describe the use of SD-OCT for the evaluation of preoperatively disrupted IS/OS junctions before and at various time points during the first 24 months after surgery. In this study, patients with a preoperatively intact IS/OS junction were excluded because we previously reported that most of these patients acquire a sufficient visual re- 
covery of nearly $20 / 20$ vision by the time of a one-year follow-up examination [8]. Although the results of the present study were inferior to these previously reported results, the present study demonstrated that patients with disrupted IS/OS junctions after ERM surgery could achieve anatomic and functional recovery even after 1 year postoperatively.

In our study, 7 patients (41.2\%) had IS/OS junctions with a normal appearance at the time of a 24-month follow-up examination. Furthermore, BCVA improvement after surgery in these eyes was significantly better than that of the remaining 10 eyes, which showed a persistently disrupted IS/OS junction. In a previous study, we reported that the disruption of the IS/OS junction, namely photoreceptor damage, as identified preoperatively may be caused by mechanical traction induced by the ERM and may be irreversible even after successful removal of the ERM. However, in fact, such mechanical damage could be completely restored after more than 1 year, enabling functional improvement, although not all of the cases were able to achieve this recovery.

We classified the preoperatively disrupted IS/OS junctions into two groups: LMH type and S type. LMH type eyes, as opposed to $S$ type eyes, tended to exhibit persistently disrupted IS/OS junctions at 24 months after surgery. Previous reports have indicated that photoreceptor layer defects were seen in $29 \%$ of cases with non-fullthickness macular holes [10], and these characteristics represent the long-term findings of LMHs [11]. Regarding the mechanisms of LMH, several hypotheses (mainly the following three) have been proposed, including centripetal traction with the ERM [12], anteroposterior traction resulting in intraretinal dissociation [10], and degeneration associated with chronic macular edema [13]. We speculated that such chronic cystoid macular edemas may lead not only to LMH but also to photoreceptor layer disruption that is possibly dependent on chronicity (in other words, the duration of symptoms before the surgery). Furthermore, the macular morphology of LMH after surgery has not been previously described. In our study, eyes with a larger preoperative length of the IS/OS junction defect among LMH type eyes tended to show a persistently disrupted IS/OS junction after surgery, resulting in a poorer improvement in visual acuity. Whether these disruptions are permanent is uncertain because 2 eyes with a shorter defect exhibited complete IS/OS junction recovery after 24 months. Longer follow-up investigations are warranted in the future.

On the other hand, S type eyes showed diffuse hyporeflectivity of the IS/OS junction that gradually recovered after surgery and resumed a normal appearance in more than half of the $S$ type eyes. The diffuse traction of ERM might produce a distortional force in the photoreceptor layer, resulting in the disarrangement of the photoreceptor cells and causing the $S$ type disruption. By removing the ERM traction, the morphology of the IS/OS junction might gradually recover, with hyperreflectivity observed during SD-OCT. Since $4 \mathrm{~S}$ type eyes continued to exhibit a partially hyporeflective IS/OS junction, more than 2 years may be required for complete recovery. However, in both LMH and S type eyes, the delayed recovery of the IS/ OS junction might influence the photoreceptor functional outcome, resulting in unsatisfactory visual outcomes even if complete anatomic recovery occurs in the future.

We believe that examining the preoperative cataract severity in this study would not add much new data, compared with studies where the lenses are not removed routinely during ERM studies. The baseline cataract status was relatively mild to moderate. We assessed the changes in BCVA by comparing the BCVA at 12 and 24 months and improvement in visual acuity for 24 months, at which times cataract surgery would likely have a minimal influence on the outcomes. Furthermore, a benefit of this study, in which cataract removal was performed at the time of the surgery, is that it removes the additional variable of a progressive cataract confounding the final visual outcome.

Regarding ERM surgery, deciding when to perform a vitrectomy can be difficult. The Blue Mountains Eye Study, which examined the natural history of ERM, reported that most ERM cases showed no progression for more than 5 years [14]. A recent study also reported that visual acuity was relatively conserved in the presence of LMH [15]. Therefore, macular surgery in patients with ERM might be delayed even if accompanied by $\mathrm{LMH}$, which was differentiated from full-thickness macular hole surgery. However, as initially described, although patients with disrupted IS/OS junctions after ERM surgery were able to experience visual recovery even after more than 1 year postoperatively, their outcomes were still inferior to those with preoperative intact IS/OS junctions. Therefore, it might be better to schedule vitrectomy surgery while the IS/OS junction remains intact. If the preoperative IS/OS junction has already been disrupted, the surgery should be scheduled as early as possible, since chronic LMH and a longer diffuse traction induced by ERM might delay IS/OS junction recovery and produce permanent impairment of the photoreceptor layer, resulting in further disturbances of visual improvement. 
A limitation of this study was its small sample size, consisting of only 17 patients. A larger sample size is needed to confirm the results of our study. Furthermore, this study included subjective evaluations of the SD-OCT findings. Although SD-OCT allows for precise visualization of the intraretinal morphology, such as the ELM and the photoreceptor layer, the development of an imaging analysis, especially one that enables objective measurements, is needed in the future.

In conclusion, preoperatively disrupted IS/OS junctions were able to acquire anatomic and functional recov- ery even after more than 1 year postoperatively. However, sufficient visual improvement may be difficult in eyes with persistently disrupted IS/OS junctions at 24 months, especially LMH type eyes.

\section{Disclosure Statement}

The authors have no proprietary interest in the materials used in this study.

\section{References}

1 Machemer R: The surgical removal of epiretinal macular membranes (macular puckers) (author's transl). Klin Monatsbl Augenheilkd 1978;173:36-42.

2 Rice TA, De Bustros S, Michels RG, Thompson JT, Debanne SM, Rowland DY: Prognostic factors in vitrectomy for epiretinal membranes of the macula. Ophthalmology 1986; 93:602-610.

-3 Wong JG, Sachdev N, Beaumont PE, Chang AA: Visual outcomes following vitrectomy and peeling of epiretinal membrane. Clin Experiment Ophthalmol 2005;33:373-378.

$\checkmark 4$ Trese MT, Chandler DB, Machemer R: Macular pucker. 1. Prognostic criteria. Graefes Arch Clin Exp Ophthalmol 1983;221:12-15.

5 Michalewski J, Michalewska Z, Cisiecki S, Nawrocki J: Morphologically functional correlations of macular pathology connected with epiretinal membrane formation in spectral optical coherence tomography (SOCT). Graefes Arch Clin Exp Ophthalmol 2007;245:1623-1631.
6 Suh MH, Seo JM, Park KH, Yu HG: Associations between macular findings by optical coherence tomography and visual outcomes after epiretinal membrane removal. Am J Ophthalmol 2009;147:473-480.e3.

-7 Mitamura Y, Hirano K, Baba T, Yamamoto S: Correlation of visual recovery with presence of photoreceptor inner/outer segment junction in optical coherence images after epiretinal membrane surgery. $\mathrm{Br} \mathrm{J}$ Ophthalmol 2009;93:171-175.

$\checkmark 8$ Inoue M, Morita S, Watanabe Y, Kaneko T, Yamane S, Kobayashi S, Arakawa A, Kadonosono K: Inner segment/outer segment junction assessed by spectral-domain optical coherence tomography in patients with idiopathic epiretinal membrane. Am J Ophthalmol 2010;150:834-839.

-9 Inoue M, Morita S, Watanabe Y, Kaneko T, Yamane S, Kobayashi S, Arakawa A, Kadonosono K: Preoperative inner segment/outer segment junction in spectral-domain optical coherence tomography as a prognostic factor in epiretinal membrane surgery. Retina 2011; 31:1366-1372.

10 Michalewska Z, Michalewski J, Odrobina D, Nawrocki J: Non-full-thickness macular holes reassessed with spectral domain optical coherence tomography. Retina 2012;32: 922-929.
11 Casparis H, Bovey EH: Surgical treatment of lamellar macular hole associated with epimacular membrane. Retina 2011;31:17831790.

12 Allen AW Jr, Gass JD: Contraction of a perifoveal epiretinal membrane simulating a macular hole. Am J Ophthalmol 1976;82: 684-691.

13 Gass JD: Lamellar macular hole: A complication of cystoid macular edema after cataract extraction: a clinicopathologic case report. Trans Am Ophthalmol Soc 1975;73:231-250.

14 Fraser-Bell S, Guzowski M, Rochtchina E, Wang JJ, Mitchell P: Five-year cumulative incidence and progression of epiretinal membranes: the Blue Mountains Eye Study. Ophthalmology 2003;110:34-40.

15 Theodossiadis PG, Grigoropoulos VG, Emfietzoglou I, Nikolaidis P, Vergados I, Apostolopoulos M, Theodossiadis GP: Evolution of lamellar macular hole studied by optical coherence tomography. Graefes Arch Clin Exp Ophthalmol 2009;247:13-20. 